\title{
Addressing Human Factors and Ethics in the design of 'Future Work' and Intelligent Systems for use in Financial Services - person centered operations, Intelligent Work \& the Triple Bottom Line
}

\author{
Joan Cahill[10000-0001-6944-744X], Vivienne Howard ${ }^{1}$, Yufei Huang ${ }^{2}$, Junchi Ye, Stephen \\ Ralph $^{3}$, Aidan Dillon ${ }^{3}$ \\ ${ }^{1}$ School of Psychology, Trinity College Dublin, Dublin, Ireland \\ 2 Trinity School of Business, Trinity College Dublin, Dublin, Ireland \\ ${ }^{3}$ Zarion Ltd. \\ cahilljoetcd.ie
}

\begin{abstract}
.
New technologies are being introduced to support the future of work in Financial Services. Such technologies should enable work that is smart, healthy, and ethical. This paper presents an innovative and blended methodology for supporting the specification of these future 'intelligent work' technologies from a human factors and ethics perspective. The methodology involves the participation of a community of practice and combines traditional stakeholder evaluation methods (i.e., interviews, workshops), with participatory foresight activities, participatory co-design, and data assessment.
\end{abstract}

Keywords: Human Factors, Ethics, Emerging Methods, The Future of Work, Intelligent Work, Healthy Work, Triple Bottom Line, Operations Management

\section{Introduction}

Work represents for an enterprise a significant cost in resource. Operational efficiencies are critical to the business model and a fundamental key performance indicator (KPI) for all stakeholders. However, as stated by Elkington (2019), in the 'Triple bottom line' accounting framework, human activity should not compromise the longterm balance between the economic, environmental, and social pillars [1]. Further, as defined by the tripartite labor collaboration work (and work activity) should be designed to benefit all stakeholders - including employers, employees, and society [2]. Financial institutions are utilizing new technologies (including machine learning and artificial intelligence) which enables them to manage their business processes, their workforce, and customer relationships. The technologies can be classified into four overall types - Robotic Process Automation (RPA) technologies, Business process management (BPM) technologies, Digital Process Automation (DPA) technologies and Dynamic case management (DCM) technologies. Overall, the focus is on stream- 
lining business processes, optimizing resources, and enhancing productivity and efficiency. Although the benefits in relation to productivity/efficiency have been demonstrated, the adoption of these new technologies has been slow. In many cases, the barriers to adoption are not well researched/understood. Further, these technologies have not been considered from the perspective of the human role in the workplace and worker wellbeing. Workers have concerns about how these technologies will transform their job (including how work is assigned and assessed) and the experience of work (i.e., location of work, social interaction, workload, monitoring). The COVID 19 Pandemic and largescale transition to remote work/operations, has underscored the human and ethical issues surrounding work and workforce surveillance, issues pertaining to social isolation, and the impact on team interactions (including activities such as mentoring and formal and informal teamwork).

The 'Intelligent Work' project investigates how automation, artificial intelligence technologies and workers can work together in a more efficient, intelligent, and humane way - to improve worker wellbeing along with the company's long-term revenue. This research is part of an academic and industry collaboration between researchers at Trinity College Dublin Ireland and Zarion Ltd. The research is funded by Enterprise Ireland (Irish government agency), as part of the Innovation Partnership Program (IPP).

This paper reports on the innovative methodology used in this project to support the specification 'intelligent work' and allied technologies from a human factors and ethics perspective. First, a background to relevant concepts and methodologies is provided. The methodological approach is then introduced. A short overview of the emerging intelligent work concept is presented. The methodology is then discussed, and some conclusions drawn.

\section{Background}

\subsection{Operations Management, Healthy Work \& Workplace Wellbeing}

Operational management refers to the ways in which a business manages the resources responsible for delivering work. Typically, operations management focuses on the business processes and technologies required to achieve the economic goals for the company. Often the 'human factor' and the relationship between worker wellbeing and system design is not considered. The business case for investing in worker wellbeing is well documented [3]. Poor worker wellbeing has a cost implication. For example, costs associated with reduced productivity/delays, reduced worker motivation and poor-quality work, staff retention, sick leave, errors, and poor customer service/customer retention.

New human centered business practices/operations practices are now being introduced. Such practices focus on fostering and maintaining a healthy workforce. Underpinning these approaches is the recognition that work is part of our wellbeing and a key driver of health. To this end, new work management systems and technologies 
are addressing how work is managed, the experience of work and the management of the home/work interface. This is particularly evidenced in healthcare and aviation [4].

Workers are not immune from common mental health problems such as anxiety and depression. At any given time, up to 18 per cent of the working age population has a mental health problem [5]. The level of control that an individual has over their work is a key factor for psychological health. As proposed in the 'Job Design Model' (JCM) job features such as skill variety, task identity, task significance, feedback, and task autonomy are enriching and thereby motivating, characteristics of work [6].

The World Health Organisation (WHO) proposes a model of the healthy workplace in which both physical and psychosocial risks are managed [7]. Stress Management Initiatives' (SMI) and 'Workplace Wellbeing Programs' (WWP) address workplace stress and overall health and wellbeing in the workplace [8]. Some wellness programs deploy corporate wellness self-tracking technologies (CWST) [9]. Workers are invited to measure and manage their own health, to improve their wellbeing, while also enhancing productivity, engagement, and performance. This approach is not uncontroversial. Some argue that CWST conflates work and health [10] and has the potential to increase worker anxiety levels [11].

\subsection{Stakeholder Evaluation \& Human Factors Methods}

As defined in ISO 6385 [12], the discipline of human factors (HF) refers to 'the practice of designing products, systems, or processes to take proper account of the interaction between them and the people who use them' (2016). Human factors approach follows a 'socio-technical systems design' perspective. Central to this is the recognition of the interaction between people/behavior, technology/tools, work processes, workplace environments and work culture [13]. 'Stakeholder evaluation' is the gold standard for human factors action research pertaining to new technology development. The objective is to elicit the perspectives of those who have a "stake" in implementation/change. Stakeholder evaluation methods seek to involve the participation of both internal and external stakeholders. Internal stakeholders (IS) include the project team. This composition of the internal team can vary but typically includes product owners/managers, designers, software developers and business analysts. In some cases, it can also include human factors researchers and ethicists. External stakeholders (ES) refer to those stakeholders who either who are users of the technology either directly or indirectly (i.e., financial services employees working in team members, team supervisor, operations management and leadership roles, and customers of the financial services company) and those who procure the technology (i.e., financial services company). As outlined by Cousins (2013) and Wenger (1999) [14, 15], the 'Community of Practice' is the shared space in which both IS and ES come together to ideate, define, develop and evaluation the proposed solution. Human Factors action research methods are commonly used to support this process. Typically, this involves the use of Ethnographic approaches [16] such as user interviews and stakeholder workshops. Both personae-based design [17] and scenario-based design [18] methodologies are also used. The concept of 'stakeholder participation' is a critical feature of stakeholder evaluation research. As defined by Bødker (1995), design 
happens 'with' stakeholders, and not simply 'for' stakeholders [19]. Participatory activities can include roleplay, stakeholder ideation workshops and participatory codesign and evaluation [19].

\subsection{Ethics \& New Technology Development}

New technologies have the potential to deliver benefits. However, such technologies are inherently uncertain. As part of new product development, researchers must consider and evaluate the human and ethical implications of things which may not yet exist and/or things have potential impacts which may be hard to predict [20]. Reijers et al (2017) provide an overview of the different formats in which ethics analysis in technology development take many forms [21]. Brey (2017) classifies five sets of ethical impact assessment approaches. This includes generic approaches, anticipatory/foresight approaches, risk assessment approaches, experimental approaches, and participatory/deliberative ethics approaches [22]. Some researchers have combined different approaches. Cotton (2014) combines participatory/deliberative ethics approaches and stakeholder approaches [23]. Cahill (2020) argues that human factors and ethical issues must be explored in an integrated way [24]. The 'Human Factors \& Ethics Canvas' introduced by Cahill combines ethics and HF methods, particularly around the collection of evidence using stakeholder evaluation methods [24].

\section{$3 \quad$ Research Project \& Methodology}

\subsection{Introduction}

As indicated in Figure 1 below, the collection of evidence follows from a sociotechnical framework - involving eliciting and analyzing data about the relationships between certain structuring elements of the 'socio-technical system'. Key elements include the work itself (both transactional work and knowledge work), the individuals/people and teams performing the work, the organization, and the customer. So conceived, future automation and AI/ML intelligence will change these relationships, leading to different outcomes at an economic, ecological, and societal level (i.e., triple bottom line). Critically, this automation/technology has meaning in the context of organization specific business process and associated task workflows, organizational culture, the working environment, and regulation. 


\section{High Level Intelligent Work (IW) Concept}

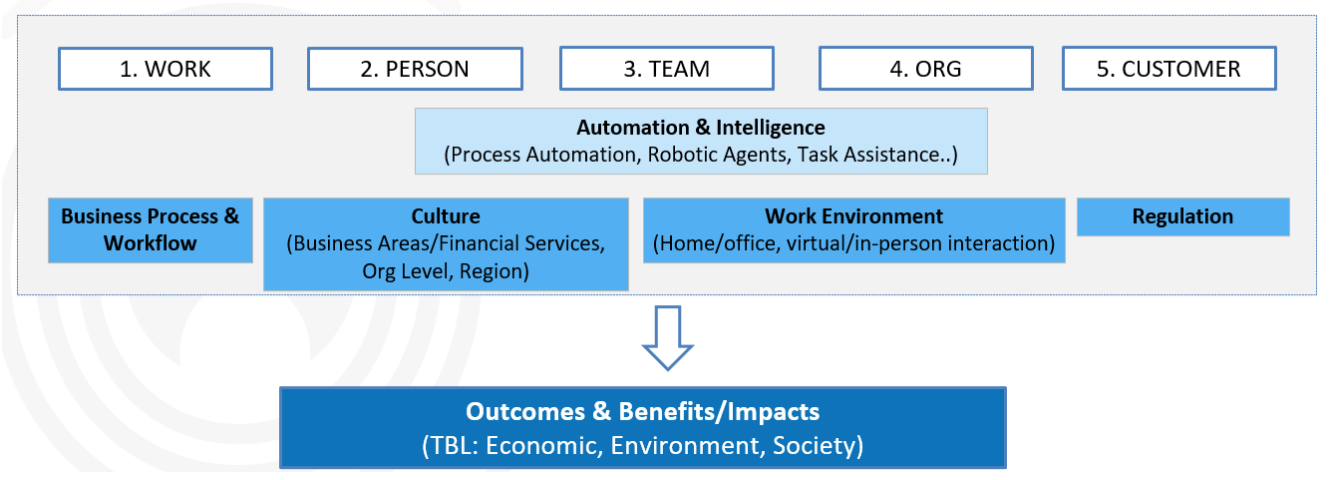

Fig. 1. Socio-technical Picture

The human factors approach adopted involves building an evidence map [25] in relation to requirements for the proposed technologies, the human factors and ethical issues pertaining to the introduction of these technologies, and the business case for these technologies. In relation to the business case, this involved investigating outcomes at an (1) organizational level (i.e., profit, productivity, employee retention), a (2) work/business process level (i.e., productivity and teamwork), (3) a worker level (i.e., job satisfaction, job engagement, wellbeing in work, trust, workload, burnout etc) and (4) a customer level (i.e., customer satisfaction, perception of brand and customer retention).

The specific methodology combined traditional human factors action research methods (i.e., interviews, workshops), with participatory foresight activities, participatory co-design and evaluation activities, and data assessment. Table 1 below provides an overview of the different human factors action research and business analysis methods used.

Table 1. Overview of Research Methods Used

\begin{tabular}{lll}
\hline$\#$ & Method & Details \\
\hline 1 & Interviews & Product team interviews/IS $(\mathrm{N}=2)$ \\
& & Interviews with Zarion staff/IS $(\mathrm{N}=6)$ \\
& Interview with ends users/ES (N=3) \\
& Product demonstration and review workshop (work- \\
& shop 1/IS, N=4) \\
& Modelling the proposed IW concept workshop (work- \\
& shop 2/IS, N=7) \\
& Evaluating the proposed IW concept workshop (work- \\
& shop 3/IS, N=7) \\
& Using Data workshop (workshop 4/IS, N=10) \\
& Business Case workshop (workshop 5/IS (N=10) \\
& Implementation, Ethics \& Acceptability workshop
\end{tabular}




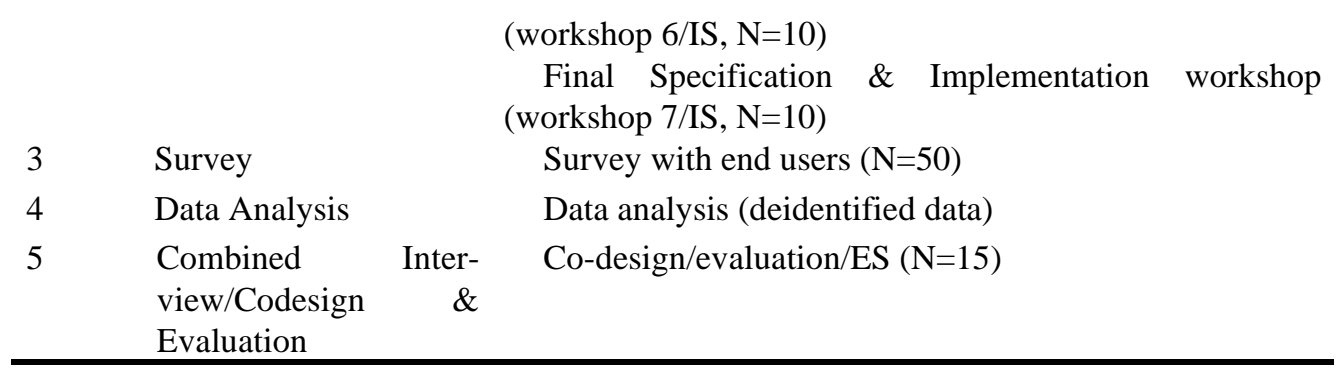

Overall, eight phases of research involving the participation of both internal stakeholders (IS) and external stakeholders (ES) was undertaken. The details of these are as defined in Table 2 below. As the research progressed, the findings of each phase were triangulated, to further develop and validate the evidence map. The study was conducted in accordance with the Declaration of Helsinki, and the protocol was approved by the Ethics Committee of the School of Psychology, Trinity College Dublin. All field research conducted online in accordance with COVID 19 health and safety guidelines - as defined by the Health \& Safety Authority, Ireland), and the definition of safe data collection, as defined by the School of Psychology, Trinity College Dublin.

Table 2. Overview of Research Stages, Methods \& Outputs

\begin{tabular}{|c|c|c|c|}
\hline \# & Stage & Methods \& Participants & Output \\
\hline 1 & $\begin{array}{l}\text { Existing product re- } \\
\text { view }\end{array}$ & $\begin{array}{l}\text { Product team interviews/IS } \\
(\mathrm{N}=2) \\
\text { Product demonstration and } \\
\text { review workshop (workshop } \\
\text { 1/IS, } \mathrm{N}=4) \text { : }\end{array}$ & $\begin{array}{l}\quad \text { Product } \\
\text { scription } \\
\text { model }\end{array}$ \\
\hline 2 & Preliminary human & HFEC Evaluation/IS (N=2) & Product Review \\
\hline & $\begin{array}{l}\text { factors and ethics as- } \\
\text { sessment }\end{array}$ & $\begin{array}{c}\text { Personae \& Scenarios } \\
\text { Specification/IS }(\mathrm{N}=2)\end{array}$ & $\begin{array}{l}\text { Personae and } \\
\text { scenarios. }\end{array}$ \\
\hline 3 & $\begin{array}{l}\text { New Product Idea- } \\
\text { tion }\end{array}$ & N/A & $\begin{array}{l}\text { Preliminary IW } \\
\text { Concept Specifi- } \\
\text { cation } \\
\text { Definition of } \\
\text { states. }\end{array}$ \\
\hline 4 & $\begin{array}{l}\text { Mapping the prob- } \\
\text { lem space \& further } \\
\text { specification/validation }\end{array}$ & $\begin{array}{l}\text { Interviews/IS }(\mathrm{N}=6) \\
\text { Interview/ES(N=3) } \\
\text { Modelling the proposed IW }\end{array}$ & $\begin{array}{l}\text { Field research } \\
\text { findings/evidence } \\
\text { map }\end{array}$ \\
\hline & $\begin{array}{l}\text { of Concept \& Re- } \\
\text { quirements }\end{array}$ & $\begin{array}{l}\text { concept workshop (workshop } \\
\text { 2/IS, } N=7 \text { ) } \\
\quad \text { Survey/ES }(\mathrm{N}=50)\end{array}$ & $\begin{array}{l}\text { Preliminary IW } \\
\text { Concept Specifi- } \\
\text { cation }\end{array}$ \\
\hline 5 & $\begin{array}{l}\text { Prototype } \\
\text { ment \&evelop- } \\
\text { views/Codesign }\end{array}$ & $\begin{array}{l}\text { Evaluating the proposed } \\
\text { IW concept workshop (work- } \\
\text { shop } 3 / \mathrm{IS}, \mathrm{N}=7 \text { ) }\end{array}$ & $\begin{array}{r}\text { Prototype De- } \\
\text { velopment } 1\end{array}$ \\
\hline
\end{tabular}




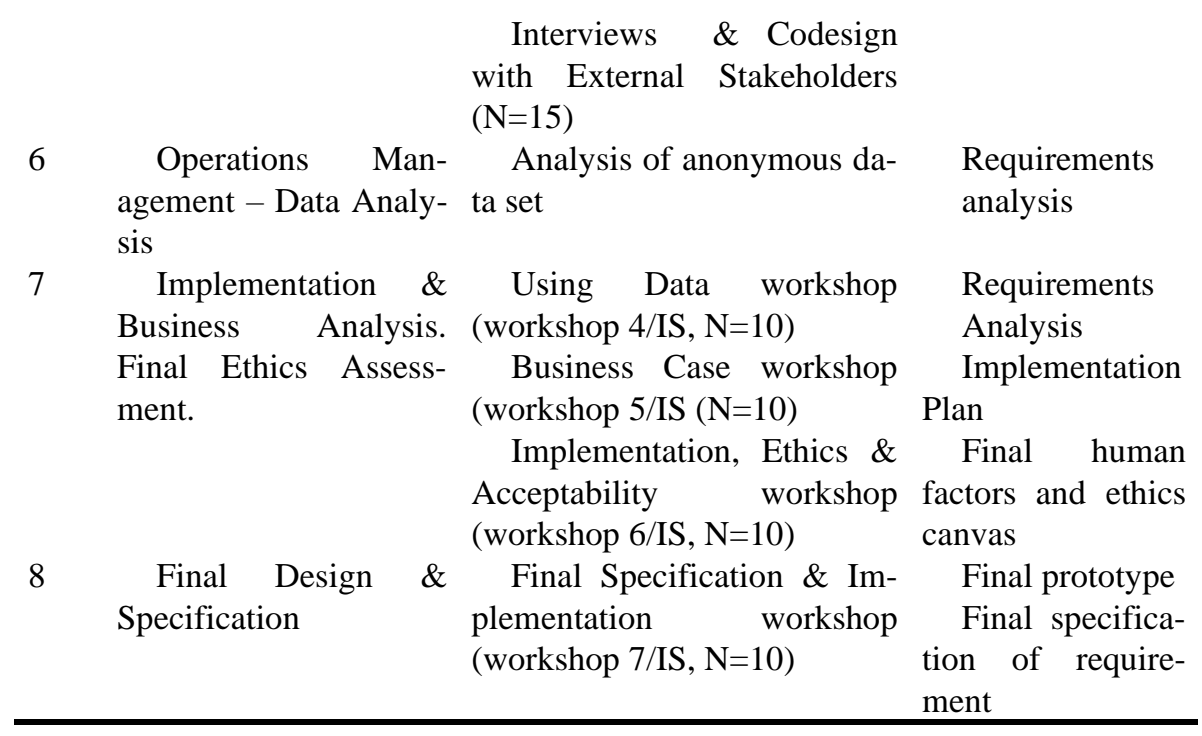

\section{Overview of Emerging Concept}

The vision is to advance technology which functions as a 'balance score card' [25] linked to the 'Triple Bottom Line' [1]. The focus is on enabling/augmenting people as opposed to health monitoring. Corporate wellness approaches such as the provision of healthy food and free/subsidized access to wellness activities (i.e., yoga, mindfulness, exercise, and stress management classes) are not enough. Healthy work concepts need to be embedded in how work is planned/allocated, carried out, monitored, and evaluated/assessed. As such, healthy work underpins intelligent work. The proposed technology will enable 'intelligent work' through the application of AI/ML, which enables healthy work allocation and monitoring - balancing different perspectives and needs - the work, the person, the team, the customer, and business value. Intelligent assistants function as supportive team members - augmenting and transforming all roles, including team members, team supervisors, operations managers, and the customer. Critically, the system supports 'coaching' of team members and worker selfregulation and self-management of work.

\section{Discussion: Emerging Methods \& Innovation}

The methodologies adopted in this project emerged out of the diverse and multidisciplinary skillset of the 'internal team'/IS. The IS comprised two organizational groups - (1) a product development team from a software development company advancing future work technologies (Zarion Ltd), and (2) a multi-disciplinary research team from Trinity College Dublin - comprising human factors, health psychology and ethics researchers from Trinity School of Psychology, and operations 
management and data scientists from Trinity School of Business. As such, the composition of the IS enabled a blending of different methodological approaches and allied technology ideation, development, and evaluation methodologies to the identification of user requirements for the proposed intelligent work system.

The research methodology blends several established and innovative human factors and ethics design and assessment methods. The emerging evidence map reflected the iterative set of requirements which emerged from these different activities.

In terms of established methods, several qualitative human machine interaction (HMI) design methods were combined to supports needs analysis and requirements specification. This includes 'personae-based design', 'scenario-based design' and 'participatory design'. Survey methods were also used to elicit requirements. The survey analysis provided a complementary picture to the interview and codesign/evaluation activities.

In terms of more innovative methods - this includes the integration and application of the 'Human Factors \& Ethics Canvas' (HFEC) (Cahill, 2020), into the high-level methodology. Each of the seven stages of the HFEC was populated, with the emerging evidence picture. In relation to stage 3 (personae and scenarios), each personae and scenario was defined in relation to specific IW states. This included states to be achieved (i.e., wellness, flow, engagement), states to be managed/mitigated (i.e., stress, overwork, poor teamwork) and states to be avoided (i.e., burnout, poor interaction with team or customer, errors, and objectification of worker/over monitoring). Personae and scenarios were defined for both workers and customers. In addition, states were defined in relation to the process, the organizational culture, and the business/organization (i.e., profit, customer retention, growth etc). This enabled an integration of both human factors and business objectives, linking to the underpinning value/benefits assessment approach (i.e., triple bottom line). This was further progressed in Stage 4 of the HFEC - the assessment of benefits, outcomes, and impact. This focus on stakeholders and assessing needs/benefits is central to participatory foresight activities.

In addition, the data points associated with evaluating states at different levels (actors, process, organization etc) were defined. This enabled a bridge between human factors/ethics research, and the advancement of the product technical architecture. Further, it set a high-level remit for the role of this future IW system in terms of collecting and evaluating data and allied automation, artificial intelligence and machine learning functions.

A further innovation was the identification of future system requirements based on an analysis of operations management data at an insurance company. The anonymous data set (total of 117,452 records) was interrogated to understand and identify strategies for better work allocation and management and associated requirements for 'intelligent work' system. The data set pertained to operational performance at an insurance company over a fixed time-period. The data was analyzed at three levels - (1) activity/claims level, (2) individual level, and (3) team level. In relation to (1), this resulted in insight pertaining to the relationship between activity complexity and claims productivity (no of claims processed) with specific insights pertaining to the relationship between activity complexity and individual and team workloads. In rela- 
tion to (2) this resulted in insights in relation to activity complexity and individual productivity, with specific insights in relation to the relationship between activity complexity and workload, work diversity, and teamwork rate. Lastly, in relation to (3), this resulted in insights pertaining to the relationship between individual productivity and team productivity, with specific insights pertaining to the relationship between productivity and individual/team location, team size, days worked and work diversity.

A key strand of this research activity involved understanding the motivations, enablers, and barriers to implementation. This links to the sixth stage in the Human Factors \& Ethics Canvas (Cahill, 2020). Issues pertaining to implementation were addressed during interviews with $\mathrm{E} / \mathrm{S}$, co-design/evaluation sessions with $\mathrm{E} / \mathrm{S}$ and implementation workshops with I/S. As part of this, storytelling and narrative techniques were used to capture the future 'story' and/or 'implementation' of the technology. The future 'implementation story' had a high-level tagline, a plot, a context/setting, key characters, and an ending. Participants were invited to consider two taglines and associated plots, which reflected a summary of the research findings. These were: (1) "Move from task to people centric", and (2) "The organization gets the right balance, the customer get the right balance and the people get the right balance". Storytelling was considered an accessible and user-friendly approach to product ideation, requirements specification and requirements evaluation. Overall, this storytelling approach enabled a synthesis and integration of different types of requirements (i.e., need, acceptability, ethics, software role, busines value and implementation), from different perspectives (i.e., human factors, ethics, operations management, and business case/benefits).

Some limitations should be noted. Observational research at financial services companies was planned, but not possible during the COVID 19 pandemic. Such research might have substantiated some of the issues around work practices, use of technology and work culture which arose in user interviews. Although three phases of combined interviews and co-design/evaluations were undertaken, the numbers in each phase were small $(\mathrm{N}=5$ in each phase, total number: $\mathrm{N}=15)$. Further a small number of participant's completed the survey $(\mathrm{N}=50)$. The operations management dataset reflected work activity that was managed without formal work allocation/process management software. Further research might involve the analysis of operations management activity where a basic and/or intelligent work allocation software platform is used.

Further research is planned. This research has resulted in a proof of concept for the future work system. To date, research is mostly conceptual. The next phases of research will involve simulation of a small set-of scenarios with accompanying intelligent work software, to demonstrate and evaluate the human factors and business benefits of embedding healthy, smart, and ethical work concepts in new 'intelligent work' technologies. 


\section{Conclusion}

New intelligent work technologies should support enable work that is smart, healthy, and ethical. This involves moving beyond simply process automation and robotic team members. Technologies should augment all human actors, promote teamwork behaviors, and ensure that human actors can self-manage and monitor their own performance. In so doing future 'intelligent work' systems should deploy artificial intelligence (AI) and Machine Learning (ML) technologies in a human centered and ethical manner.

Disorganized, fragmented, imbalanced, and unfair workloads can impact on worker productivity, engagement, and 'the flow state'. Technology may not be the barrier here - when there is insufficient information and poor teamwork, productivity significantly decreases.

The methodologies used in this project enable the active translation of human factors and ethical principles along with stakeholder needs, into the product concept and design execution. Personae/scenarios are useful in relation to considering and documenting the needs/perspectives of different stakeholders and adjudicating between conflicting human factors and ethical goals/principles. Co-design methods are useful for product ideation and eliciting feedback about ethical issues along with implementation barriers. The use of a 'Community of Practice' has proven very beneficial. It is critical to engage both internal and external stakeholders in the human factors and ethics specification and validation of the proposed technologies, and analysis of implementation requirements, barriers, and enablers.

\section{References}

1. Elkington, John. Cannibals with forks: the triple bottom line of 21 st century business. Oxford: Capstone. ISBN 9780865713925 . OCLC 963459936 (1999).

2. Sengenberger $\mathrm{W}$ The international labour organization: goals, functions and political impact. Friedrich Ebert Stiftung, Berlin. 2013.

3. Bevan S (2010) The business case for employee health and wellbeing: A report prepared for investors in people UK. Available at: http://workfoundation.org/assets/docs/publications/245_iip270410.pdf

4. Cahill, J.; Cullen, P.; Anwer, S.; Gaynor, K.; Wilson, S. (2020). The Requirements for New Tools for Use by Pilots and the Aviation Industry to Manage Risks Pertaining to Work-Related Stress (WRS) and Wellbeing, and the Ensuing Impact on Performance and Safety. Technologies (2020), 8, 40. https://www.mdpi.com/2227-7080/8/3/40. https://doi.org/10.3390/technologies 8030040

5. National Institute for Health \& Care Excellence (NICE, 2011). Mental Wellbeing at Work. Retrieved from: https://www.nice.org.uk/guidance/ph22

6. Hackman, J.R. and Oldham, G.R. (1976), "Motivation through the design of work: test of a theory", Organizaional Behavior and Human Performance, Vol. 16 No. 2, pp. 250-279.

7. The World Health Organisation (WHO). Healthy workplace model. Available at: https://www.who.int/occupational_health/healthy_workplace_framework.pdf

8. Tetrick, Lois and Winslow, Carolyn, Workplace Stress Management Interventions and Health Promotion. Annual Review of Organizational Psychology and Organizational Be- 
havior, Vol. 2, Issue 1, pp. 583-603, 2015, Available at http://dx.doi.org/10.1146/annurevorgpsych-032414-111341

9. Till C, Petersen A, Tanner C, Munsie M. Creating "automatic subjects": Corporate wellness and self-tracking. Health: An Interdisciplinary Journal for the Social Study of Health, Illness \& Medicine. 2019;23(4):418.

10. Hull, G. \& Pasquale, F. Toward a critical theory of corporate wellness. BioSocieties 13(1): 190-212. 2018.

11. Moore, P \& Robinson, A. The quantified self: What counts in the neoliberal workplace. New Media \& Society 18(11): 2774-2792. 2016.

12. International Standards Organisation (ISO). 2020. Standard 6385 Available from: https://www.iso.org/standard/63785.html.

13. Baxter, Gordon \& Sommerville, Ian. 2011. Socio-technical systems: From design methods to systems engineering, Interacting with Computers, Volume 23, Issue 1, January 2011, Pages 4-17, https://doi.org/10.1016/j.intcom.2010.07.003

14. Cousins, J.B.; Whitmore, E.; Shulha, L. 2013. Arguments for a common set of principles for collaborative inquiry in evaluation. Am. J. Eval. 34, 7-22.

15. Wenger, E. Communities of Practice: Learning, Meaning, and Identity; Cambridge University Press: Cambridge, UK (1998).

16. Hammersley, M.; Atkinson, P. Ethnography: Principles in Practice, 3rd ed.; Routledge: London, UK, 2007.

17. Pruitt, J. \& Grudin, J. Personas: Practice and Theory. In Proceedings of the 2003 conference on Designing for user experiences (DUX '03). ACM, New York, NY, USA, 1-15. DOI=http://dx.doi.org/10.1145/997078.997089 (2003).

18. Carroll, J.M. Scenario-based design: Envisioning work and technology in system development. John Wiley and Sons, New York. (1995).

19. Bødker, S (1996). "Creating conditions for participation: Conflicts and resources in systems design". Human Computer Interaction. 11 (3): 215-236. doi:10.1207/s15327051hci1103_2.

20. Capurro, R. Digital Ethics. The Academy of Korean Studies (ed.): Civilization and Peace, Korea: Academy of Korean Studies 2010, pp. 203-214. (2009)

21. Reijers, W., Wright, D., Brey, P., Weber, K., Rodrigues, R., O'Sullivan, D., Gordijn, B.: Methods for Practising Ethics in Research and Innovation: A Literature Review, Critical Analysis and Recommendations. Science and Engineering Ethics (2017).

22. Brey, P. Ethics of Emerging Technologies. In S. O. Hansson (Ed.), Methods for the Ethics of Technology. Rowman and Littlefield International (2017).

23. Cotton, M. Ethics and Technology Assessment: A Participatory Approach. Berlin: SpringerVerlag (2014).

24. Cahill J. (2020) Embedding Ethics in Human Factors Design and Evaluation Methodologies. In: Duffy V. (eds) Digital Human Modeling and Applications in Health, Safety, Ergonomics and Risk Management. Human Communication, Organization and Work. HCII 2020. Lecture Notes in Computer Science, vol 12199. pp 217-227 Springer, Cham. DOI: 10.1007/978-3-030-49907-5_15

25. Miake-Lye, I.M., Hempel, S., Shanman, R. et al. What is an evidence map ? 2016. A systematic review of published evidence maps and their definitions, methods, and products. Syst Rev 5, 28 doi :10.1186/s13643-016-0204-x

26. Kaplan, Robert S.; Norton, David P. (1992-01-01). "The Balanced Scorecard-Measures that Drive Performance". Harvard Business Review (January-February 1992). Available at https://hbr.org/1992/01/the-balanced-scorecard-measures-that-drive-performance-2 